\title{
Thermo-resonance analysis of an excited graphene sheet using a new approach
}

\author{
Mohammad Malikan $^{a^{*}}$, Rossana Dimitri $^{b}$, Francesco Tornabene $^{c}$ \\ ${ }^{a}$ Department of Mechanical Engineering, Islamic Azad University, Mashhad Branch, Mashhad, Iran, \\ ${ }^{b, c}$ Department of Innovation Engineering, Università del Salento, Lecce, Italy, \\ E-mail address: mohammad.malikan@yahoo.com ${ }^{\text {a }}$, rossana.dimitri@unisalento.it ${ }^{\text {b }}$, \\ francesco.tornabene@ unisalento.it ${ }^{c}$
}

Received date: 31.08 .2018

Accepted date: 30.10 .2018

ORCID numbers of authors

0000-0001-7356-2168 ${ }^{\mathrm{a}}, 0000-0001-7153-4307^{\mathrm{b}}, 0000-0002-5968-3382^{\mathrm{c}}$

\begin{abstract}
This paper analyzes the thermo-vibration response of a graphene sheet excited with a uniform harmonic The problem is here tackled with a novel approach combined with a nonlocal strain gradient theory (NSGT), in order to include the size-dependence and the nonlocality effect on impacts. Simply-supported plates are here studied analytically, according to the Navier's method. Thus, the thermo-forced vibration equations of the problem are here written and solved numerically for graphene sheets. The accuracy of the proposed theory is checked by means of several comparative evaluations with respect to the available results from literature. Another key aspect of the works is the sensitivity of the thermo-mechanical response of the plate structures to different thermal and mechanical input parameters. This could be of great interest for design purposes for many engineering applications, including nanoelectromechanical systems (NEMS), biosensors, piezoelectric devices, biomechanical tissues, among others.
\end{abstract}

Keywords: Thermo-forced vibrations; A novel approach; Nonlocal theory of strain gradient

\section{Introduction}

Due to large variety of applications of graphene for many electronic components at the nanoscale, many studies in the literature on graphene materials have enhanced the development of lower-volume and high-speed electronic components. Graphene exhibits some physical properties usually not visible at a nanoscale level, namely, the high flexibility [1], the high thermal-mechanical resistance and elastic modulus [2]. More recently, an increased attention has been paid to graphene-based semiconductor photocatalysts [3], [4]. A graphene material is usually classified by means of a number of stacking layers, monolayer or multi-layer [5]. The use of a single-layer graphene nanoplate cannot only be a high-quality two-dimensional photocatalyst backup, but also can be a two-dimensional current circuit with a potentially significant potential for full redox and electrical properties [3]. To understand the main properties of single-layer graphene sheets, different works in literature have studied their mechanical and physical behavior. Among them, Ebrahimi and Barati [6] investigated the damped frequencies of nanoplates, according to a modified higher-order plate approach 
combined with a general NSGT. The differential quadrature (DQ) technique has been applied to get numerical outcomes for simple edges. Radic and Jeremic [7] investigated the nonlocal stability of bi-layered nano sheets resting on a polymer foundation under in-plane thermal forces. Malikan et al. [8] employed the DQ technique to examine the stability of bi-layered nano sheets exposed to in-plane thermal and shear forces bridged on the polymer substrate. In the additional works by Malikan [9],[10], the author studied a laminated plate with graphene covering, subjected to in-plane mechanical forces, based on a refined couple stress theory [9], as well as the stability of nanoplates compressed nonuniformly [10]. The stability of nanoplates embedded on a polymer substrate was also analyzed by Shahsavari et al. [11] in a hygrothermal environment, while applying different NSGTs. Additional applications of nonlocal methods can be found in literature for stability problems of graphene sheets in thermal environment [12], or frequency problems of nano sheets under axial magnetic forces [13], nonlinear natural frequencies of beams made of graded materials reinforced with nanoplatelets [14]. Moreover, Gholami and Ansari [15] studied the nonlinear vibration of composite rectangular plates reinforced with graphene platelets, through the application of the third-order shear deformation model. Further relevant works on the topic can be found in [1651], where different nanostructures have been considered for varying conditions.

Differently from the available literature on the topic, here we propose a refined plate approach with a reduced number of variables in comparison with the first-order shear deformation theory. The nanoplate is excited uniformly and harmonically under a transverse load. To model the thermo-vibration response, the nanoplate is considered in a thermal environment. The nonlocal reaction between atoms is here analyzed trough a nonlocal elasticity theory, whereby the mechanical behavior at a microscale and nanoscale accounts for the sizedependence of impacts and stiffness, in agreement with experimental evidences. Hence, the microstructural problem is here tackled with a modified couple stress method, whereas the nanostructural one is here studied accounting for the second order strain gradient term. A theoretical procedure is here proposed for the study of the thermomechanical behavior of simply supported nanoplates, while exploring the accuracy of the method and the sensitivity of the response to different input parameters.

\section{Mathematical modeling}

Fig. 1a shows an idealized and realistic model for graphene nanoplates. The plate is characterized by a length $L_{x}$, width $L_{y}$ and thickness $h$ in a Cartesian coordinate system, and it is pressured transversely by a uniform dynamic force in a thermal environment. More details about the dynamic force acting transversely on the body are depicted in Fig. 1b [52]. A novel plate model is here proposed, which assumes the following displacement field [53-59]

$$
\left\{\begin{array}{l}
U(x, y, z, t) \\
V(x, y, z, t) \\
W(x, y, z, t)
\end{array}\right\}=\left\{\begin{array}{l}
u(x, y, t)-z \frac{\partial w_{0}(x, y, t)}{\partial x} \\
v(x, y, t)-z \frac{\partial w_{0}(x, y, t)}{\partial y} \\
w_{0}(x, y, t)+A \frac{\partial^{2} w_{0}(x, y, t)}{\partial x^{2}}+B \frac{\partial^{2} w_{0}(x, y, t)}{\partial y^{2}}
\end{array}\right\}
$$




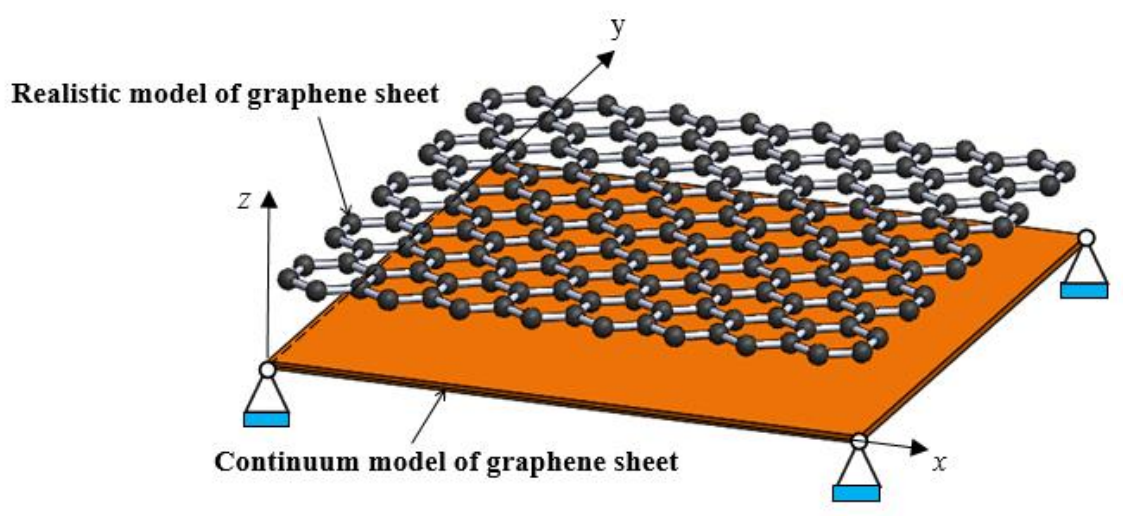

Fig.1a. Graphene nanoplate subjected to thermal environment in 3D Cartesian coordinate system

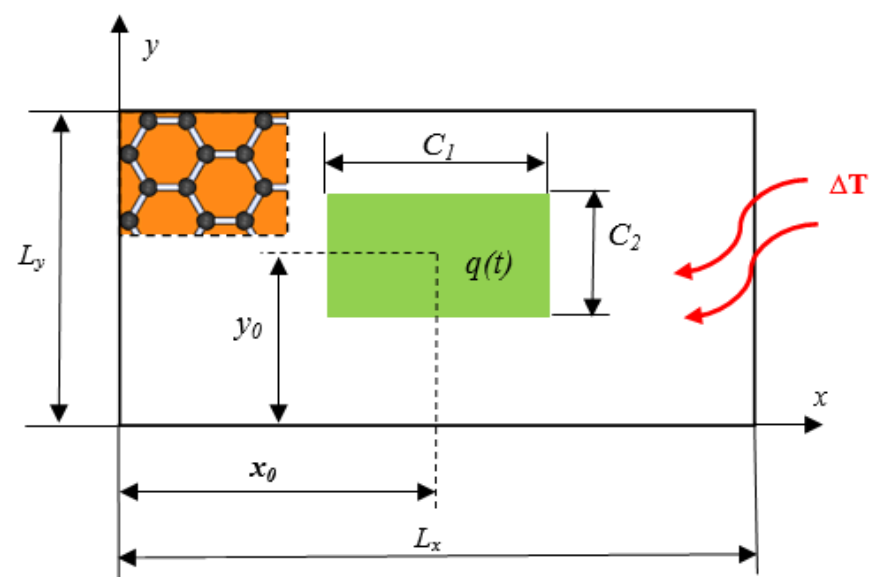

Fig.1b. The dynamic load acted harmonically and uniformly

As far as the Hamilton's approach is concerned, the potential energy of the model, $V$, is defined as [60]

$$
\delta V=\delta \int_{0}^{t}(S+\Omega-T) d t=0
$$

where $\delta \Omega$ is the variation of the external work, $\delta T$ refers to the variation of the kinetic energy and $\delta S$ is the variation of the strain energy. This last contribution is computed as

$$
\delta S=\iiint_{V} \sigma_{i j} \delta \varepsilon_{i j} d V=0
$$

Eq. (1) is substituted in the nonlinear Lagrangian strain filed, which leads to [58] 


$$
\begin{aligned}
& \left\{\begin{array}{l}
\varepsilon_{x x} \\
\varepsilon_{y y} \\
\gamma_{x z} \\
\gamma_{y z} \\
\gamma_{x y}
\end{array}\right\}= \\
& \left\{\begin{array}{l}
\frac{\partial u}{\partial x}-z \frac{\partial^{2} w_{0}}{\partial x^{2}}+\frac{1}{2}\left(A \frac{\partial^{3} w_{0}}{\partial x^{3}}+B \frac{\partial^{3} w_{0}}{\partial x \partial y^{2}}+\frac{\partial w_{0}}{\partial x}\right)^{2} \\
\frac{\partial v}{\partial y}-z \frac{\partial^{2} w_{0}}{\partial y^{2}}+\frac{1}{2}\left(A \frac{\partial^{3} w_{0}}{\partial x^{2} \partial y}+B \frac{\partial^{3} w_{0}}{\partial y^{3}}+\frac{\partial w_{0}}{\partial y}\right)^{2}
\end{array}\right. \\
& \left\{A \frac{\partial^{3} w_{0}}{\partial x^{3}}+B \frac{\partial^{3} w_{0}}{\partial x \partial y^{2}}\right. \\
& A \frac{\partial^{3} w_{0}}{\partial x^{2} \partial y}+B \frac{\partial^{3} w_{0}}{\partial y^{3}} \\
& \left(\left(\frac{\partial u}{\partial y}+\frac{\partial v}{\partial x}\right)-2 z \frac{\partial^{2} w_{0}}{\partial x \partial y}+\left(A \frac{\partial^{3} w_{0}}{\partial x^{3}}+B \frac{\partial^{3} w_{0}}{\partial x \partial y^{2}}+\frac{\partial w_{0}}{\partial x}\right)\left(A \frac{\partial^{3} w_{0}}{\partial x^{2} \partial y}+B \frac{\partial^{3} w_{0}}{\partial y^{3}}+\frac{\partial w_{0}}{\partial y}\right)\right)
\end{aligned}
$$

Furthermore, the kinetic energy is defined as $[52,60]$

$$
T=\frac{1}{2} \int_{A} \int_{-h / 2}^{h / 2} \rho(z, T)\left(\left(\frac{\partial U}{\partial t}\right)^{2}+\left(\frac{\partial V}{\partial t}\right)^{2}+\left(\frac{\partial W}{\partial t}\right)^{2}\right) d z d A=0
$$

whereas its variational form reads as follows

$$
\begin{aligned}
& \delta T=\int_{A} \int_{-h / 2}^{h / 2} \rho(z, T)\left[\left(-z^{2} \frac{\partial^{4} w_{0}}{\partial x^{2} \partial t^{2}}-z^{2} \frac{\partial^{4} w_{0}}{\partial y^{2} \partial t^{2}}-\frac{\partial^{2} w_{0}}{\partial t^{2}}-A^{2} \frac{\partial^{6} w_{0}}{\partial x^{4} \partial t^{2}}\right.\right. \\
& \left.\left.-B^{2} \frac{\partial^{6} w_{0}}{\partial y^{4} \partial t^{2}}-2 A \frac{\partial^{4} w_{0}}{\partial x^{2} \partial t^{2}}-2 B \frac{\partial^{4} w_{0}}{\partial y^{2} \partial t^{2}}-2 A B \frac{\partial^{6} w_{0}}{\partial x^{2} \partial y^{2} \partial t^{2}}\right) \delta w_{0}\right] d z d A=0
\end{aligned}
$$

The numerical terms in the Eq. (6) denote the mass moments of inertia, namely [52, 60]

$$
\left(I_{0}, I_{2}\right)=\int_{-h / 2}^{h / 2} \rho(z, T)\left(1, z^{2}\right) d z
$$

Thus, the governing equations of the problem read as follows 


$$
\begin{aligned}
& -\frac{\partial^{2} M_{x}}{\partial x^{2}}-\frac{\partial^{2} M_{y}}{\partial y^{2}}-2 \frac{\partial^{2} M_{x y}}{\partial x \partial y}+A \frac{\partial^{3} Q_{x}}{\partial x^{3}}+B \frac{\partial^{3} Q_{x}}{\partial x \partial y^{2}}+A \frac{\partial^{3} Q_{y}}{\partial x^{2} \partial y}+B \frac{\partial^{3} Q_{y}}{\partial y^{3}}+ \\
& N_{x}\left(A^{2} \frac{\partial^{6} w_{0}}{\partial x^{6}}+B^{2} \frac{\partial^{6} w_{0}}{\partial x^{2} \partial y^{4}}+\frac{\partial^{2} w_{0}}{\partial x^{2}}+2 A B \frac{\partial^{6} w_{0}}{\partial x^{4} \partial y^{2}}+2 A \frac{\partial^{4} w_{0}}{\partial x^{4}}+2 B \frac{\partial^{4} w_{0}}{\partial x^{2} \partial y^{2}}\right)+ \\
& N_{y}\left(A^{2} \frac{\partial^{6} w_{0}}{\partial x^{4} \partial y^{2}}+B^{2} \frac{\partial^{6} w_{0}}{\partial y^{6}}+\frac{\partial^{2} w_{0}}{\partial y^{2}}+2 A B \frac{\partial^{6} w_{0}}{\partial x^{2} \partial y^{4}}+2 A \frac{\partial^{4} w_{0}}{\partial x^{2} \partial y^{2}}+2 B \frac{\partial^{4} w_{0}}{\partial y^{4}}\right)+ \\
& N_{x y}\left(2 A^{2} \frac{\partial^{6} w_{0}}{\partial x^{5} \partial y}+4 A B \frac{\partial^{6} w_{0}}{\partial x^{3} \partial y^{3}}+4 A \frac{\partial^{4} w_{0}}{\partial x^{3} \partial y}+2 B^{2} \frac{\partial^{6} w_{0}}{\partial x y^{5}}+4 B \frac{\partial^{4} w_{0}}{\partial x \partial y^{3}}+2 \frac{\partial^{2} w_{0}}{\partial x \partial y}\right)- \\
& I_{2}\left(\frac{\partial^{4} w_{0}}{\partial x^{2} \partial t^{2}}+\frac{\partial^{4} w_{0}}{\partial y^{2} \partial t^{2}}\right)-I_{0}\left(\frac{\partial^{2} w_{0}}{\partial t^{2}}+A^{2} \frac{\partial^{6} w_{0}}{\partial x^{4} \partial t^{2}}+B^{2} \frac{\partial^{6} w_{0}}{\partial y^{4} \partial t^{2}}+2 A \frac{\partial^{4} w_{0}}{\partial x^{2} \partial t^{2}}+2 B \frac{\partial^{4} w_{0}}{\partial y^{2} \partial t^{2}}+\right. \\
& \left.2 A B \frac{\partial^{6} w_{0}}{\partial x^{2} \partial y^{2} \partial t^{2}}\right)=q(x, y, t)
\end{aligned}
$$

where $N_{i}, Q_{i}$, and $M_{i}(i=x, y, x y)$ denote the stress resultants, in terms of axial forces, shear forces, and moments. Constants $D_{i j}(\mathrm{i}, \mathrm{j}=1,2$, and 6$)$ and $H_{44}$ are described by

$$
\begin{gathered}
H_{44}=G h, G=\frac{E}{2(1+v)}, Q_{11}=\frac{E}{1-v^{2}}=Q_{22}, Q_{12}=\frac{v E}{1-v^{2}}, Q_{66}=G \\
D_{i j}=\int_{-\frac{h}{2}}^{\frac{h}{2}}\left(z^{2}\right) Q_{i j} d z \quad(i=1,2,6)
\end{gathered}
$$

where $v$ is the Poisson's ratio, $E$ is the Young's modulus, and $G$ is the shear modulus for isotropic graphene sheets. Some detail about the NSGT, here applied for the theoretical study, is presented in what follows $[52,58]$

$$
\begin{gathered}
\left(1-\mu_{1} \nabla^{2}\right)\left(1-\mu_{0} \nabla^{2}\right) \sigma_{i j}=C_{i j k l}\left(1-\mu_{1} \nabla^{2}\right) \varepsilon_{k l}-C_{i j k l} l^{2}\left(1-\mu_{0} \nabla^{2}\right) \nabla^{2} \varepsilon_{k l} ; \\
\mu_{0}\left(n m^{2}\right)=\left(e_{0} a\right)^{2}, \mu_{1}\left(n m^{2}\right)=\left(e_{1} a\right)^{2}, \nabla^{2}=\frac{\partial^{2}}{\partial x^{2}}+\frac{\partial^{2}}{\partial y^{2}}
\end{gathered}
$$

where, $\mu_{0}$ and $\mu_{l}$ refer to lower and higher-order nonlocal parameters, $(l)$ is the length scale parameter. The local stress resultants read

$$
\begin{gathered}
\left(N_{x}, N_{y}, N_{x y}\right)=\int_{-h / 2}^{h / 2}\left(\sigma_{x}, \sigma_{y}, \sigma_{x y}\right) d z \\
\left(M_{x}, M_{y}, M_{x y}\right)=\int_{-h / 2}^{h / 2}\left(\sigma_{x}, \sigma_{y}, \sigma_{x y}\right) z d z \\
\left(Q_{x}, Q_{y}\right)=\int_{-h / 2}^{h / 2}\left(\sigma_{x z}, \sigma_{y z}\right) d z
\end{gathered}
$$

By combining Eqs. (4), (12)-(14), the stress resultants become 


$$
\begin{aligned}
& {\left[\begin{array}{c}
N_{x x} \\
N_{y y} \\
N_{x y} \\
M_{x x} \\
M_{y y} \\
M_{x y} \\
Q_{y} \\
Q_{x}
\end{array}\right]=\left[\begin{array}{cccccccc}
A_{11} A_{12} & 0 & 0 & 0 & 0 & 0 & 0 \\
A_{21} & A_{22} & 0 & 0 & 0 & 0 & 0 & 0 \\
0 & 0 & A_{66} & 0 & 0 & 0 & 0 & 0 \\
0 & 0 & 0 & D_{11} & D_{12} & 0 & 0 & 0 \\
0 & 0 & 0 & D_{21} D_{22} & 0 & 0 & 0 \\
0 & 0 & 0 & 0 & 0 & D_{66} & 0 & 0 \\
0 & 0 & 0 & 0 & 0 & 0 & H_{44} & 0 \\
0 & 0 & 0 & 0 & 0 & 0 & 0 & H_{44}
\end{array}\right] \times} \\
& {\left[\begin{array}{c}
\frac{\partial u}{\partial x}+\frac{1}{2}\left(A \frac{\partial^{3} w_{0}}{\partial x^{3}}+B \frac{\partial^{3} w_{0}}{\partial x \partial y^{2}}+\frac{\partial w_{0}}{\partial x}\right)^{2} \\
\frac{\partial v}{\partial y}+\frac{1}{2}\left(A \frac{\partial^{3} w_{0}}{\partial x^{2} \partial y}+B \frac{\partial^{3} w_{0}}{\partial y^{3}}+\frac{\partial w_{0}}{\partial y}\right)^{2} \\
\left(\frac{\partial u}{\partial y}+\frac{\partial v}{\partial x}\right)+\left(A \frac{\partial^{3} w_{0}}{\partial x^{3}}+B \frac{\partial^{3} w_{0}}{\partial x \partial y^{2}}+\frac{\partial w_{0}}{\partial x}\right)\left(A \frac{\partial^{3} w_{0}}{\partial x^{2} \partial y}+B \frac{\partial^{3} w_{0}}{\partial y^{3}}+\frac{\partial w_{0}}{\partial y}\right) \\
-\frac{\partial^{2} w_{0}}{\partial x^{2}} \\
-\frac{\partial^{2} w_{0}}{\partial y^{2}} \\
-\frac{\partial^{2} w_{0}}{\partial x \partial y} \\
A \frac{\partial^{3} w_{0}}{\partial x^{2} \partial y}+B \frac{\partial^{3} w_{0}}{\partial y^{3}} \\
A \frac{\partial^{3} w_{0}}{\partial x^{3}+B \frac{\partial^{3} w_{0}}{\partial x} \partial y^{2}}
\end{array}\right]} \\
& -\left\{\begin{array}{l}
N^{T} \\
N^{T} \\
0 \\
0 \\
0 \\
0 \\
0 \\
0
\end{array}\right\} ; N^{0}=-N^{T}=-E h \alpha \Delta T\left(\frac{1+v}{1-v^{2}}\right)
\end{aligned}
$$

where $N^{T}$ is the axial load associated to the thermal environment, $\alpha$ is the thermal expansion of the graphene sheet, and $\Delta T=T_{2}-T_{1}$ is the temperature variation in the thickness direction. Here we set a reference value for the temperature equal to $T_{l}=300 \mathrm{~K}$. Then, we can use of Eq. 
(11) to re-formulate the stress resultants, in order to obtain their nonlocal strain gradient form, namely

$$
\begin{gathered}
\left(1-\left(\mu_{0}+\mu_{1}\right) \nabla^{2}+\mu_{0} \mu_{1} \nabla^{4}\right) M_{x}=-\left[\left(1-\mu_{1} \nabla^{2}\right)-l^{2}\left(1-\mu_{0} \nabla^{2}\right) \nabla^{2}\right]\left(D_{11} \frac{\partial^{2} w_{0}}{\partial x^{2}}+D_{12} \frac{\partial^{2} w_{0}}{\partial y^{2}}\right) \\
\left(1-\left(\mu_{0}+\mu_{1}\right) \nabla^{2}+\mu_{0} \mu_{1} \nabla^{4}\right) M_{y}=-\left[\left(1-\mu_{1} \nabla^{2}\right)-l^{2}\left(1-\mu_{0} \nabla^{2}\right) \nabla^{2}\right]\left(D_{21} \frac{\partial^{2} w_{0}}{\partial x^{2}}+D_{22} \frac{\partial^{2} w_{0}}{\partial y^{2}}\right) \\
\left(1-\left(\mu_{0}+\mu_{1}\right) \nabla^{2}+\mu_{0} \mu_{1} \nabla^{4}\right) M_{x y}=-\left[\left(1-\mu_{1} \nabla^{2}\right)-l^{2}\left(1-\mu_{0} \nabla^{2}\right) \nabla^{2}\right]\left(D_{66} \frac{\partial^{2} w_{0}}{\partial x \partial y}\right) \\
\left(1-\left(\mu_{0}+\mu_{1}\right) \nabla^{2}+\mu_{0} \mu_{1} \nabla^{4}\right) Q_{x}=\left[\left(1-\mu_{1} \nabla^{2}\right)-l^{2}\left(1-\mu_{0} \nabla^{2}\right) \nabla^{2}\right] H_{44}\left(A \frac{\partial^{3} w_{0}}{\partial x^{3}}+B \frac{\partial^{3} w_{0}}{\partial x y^{2}}\right) \\
\left(1-\left(\mu_{0}+\mu_{1}\right) \nabla^{2}+\mu_{0} \mu_{1} \nabla^{4}\right) Q_{y}=\left[\left(1-\mu_{1} \nabla^{2}\right)-l^{2}\left(1-\mu_{0} \nabla^{2}\right) \nabla^{2}\right] H_{44}\left(A \frac{\partial^{3} w_{0}}{\partial x^{2} \partial y}+B \frac{\partial^{3} w_{0}}{\partial y^{3}}\right)(20)
\end{gathered}
$$

The thermo-excited vibration equations, can be obtained with combining Eq. (16)-(20) whilst inserting them into Eq. (8).

\section{Analytical approach}

This section employs the Navier's solution in order to study the behavior of simply-supported edges and solve the harvested equations, based on the following displacement equation [52, $56]$

$$
w_{O}(x, y, t)=\sum_{m=1}^{\infty} \sum_{n=1}^{\infty} W_{O m n} \exp \left(i \omega_{n} t\right) \sin \left(\frac{m \pi}{L_{x}} x\right) \sin \left(\frac{n \pi}{L_{y}} y\right)
$$

In Eq. (21), $m$ and $n$ denote the half-wave numbers, $W_{0 m n}$ is the displacement amplitude, and $\omega_{n}$ refer to the natural frequencies. The transverse dynamic loading applied uniformly and harmonically on the structure, reads $[52,56]$

$$
\begin{aligned}
& q(x, y, t)=\sum_{n=1}^{\infty} \sum_{m=1}^{\infty} q_{m} \exp \left(i \omega_{e x} t\right) \times \sin \left(\frac{m \pi}{L_{x}} x\right) \sin \left(\frac{n \pi}{L_{y}} y\right) \\
& q_{m}=\frac{4 q_{0}}{m n} \int_{y_{0}-c_{2} / 2}^{y_{0}+c_{2} / 2} \int_{x_{0}-c_{1} / 2}^{x_{0}+c_{1} / 2} \sin \left(\frac{m \pi}{L_{x}} x\right) \sin \left(\frac{n \pi}{L_{y}} y\right) d x d y= \\
& \frac{16 q_{0}}{m n \pi^{2}} \sin \left(\frac{m \pi}{L_{x}} x_{0}\right) \sin \left(\frac{m \pi}{L_{x}} \frac{c_{1}}{2}\right) \sin \left(\frac{n \pi}{L_{y}} y_{0}\right) \sin \left(\frac{n \pi}{L_{y}} \frac{c_{2}}{2}\right)
\end{aligned}
$$


where, $q_{m}$ represents the Fourier coefficient, $q_{0}$ denotes the load amplitude, and $\omega_{e x}$ denotes the excitation frequency. The algebraic equations can be obtained by inserting Eq. (21) and (22) into thermo-excited vibration equations. Thus yields the following relation

$$
\begin{gathered}
\left([k]-\Delta r^{2}[m]\right)\left\{W_{0 m n}\right\}=\left\{\left(1-\left(\mu_{0}^{2}+\mu_{1}^{2}\right) \nabla^{2}+\mu_{0}^{2} \mu_{1}^{2} \nabla^{4}\right) q(x, y, t)\right\} \\
\Delta r=\frac{\omega_{e x}}{\omega_{n}}
\end{gathered}
$$

where, $\Delta r$ stands for the excitation-to-natural frequency ratio, $k_{i j}(i, j=1,2,3)$ and $m_{i j}(i, j=1,2$, 3 ) refer to the stiffness and mass matrixes, respectively. After the enforcement of the null value to the determinant of the matrix coefficients, and after some mathematical manipulation, we can obtain the natural frequencies of the problem. Afterwards, by substituting the numerical frequencies into the thermo-excited vibration equations of the problem, the dynamic deflections can be found.

\section{Numerical results}

This section is devoted to the preliminary check for the accuracy of the proposed approach with respect to the available theories form the literature. Table 1 compares our results, based on a One Variable FSDT (OVFSDT), with respect to several well-known references [61-63] based on the molecular dynamics (MD), or a FSDT-DQM approach. As can be observed, growing the plate's length, makes the results nearer to the MD ones. Basically, the excellent agreement between our results and predictions from the literature verifies the high accuracy of the proposed theory. Further comparative evaluations of the results can be found in Table 2, with respect to a DQM and MD approach, in terms of mechanical stability of grapheme sheets compressed biaxially, while assuming $\mu=1.81 \mathrm{~nm}^{2}, k_{s}=5 / 6, v=0.16, E=1 T P a$.

Table 1. Validation for mechanical stability of graphene sheet compressed biaxially. $\mu=1.81 \mathrm{~nm}^{2}$, $k_{s}=5 / 6, v=0.16, E=1 T P a$.

\begin{tabular}{ccccc}
\hline \multicolumn{5}{c}{ Stability load $(n N / n m)$} \\
\hline OVFSDT & FSDT-DQM & FSDT-DQM & MD results & $L_{x}=L_{y}(\mathrm{~nm})$ \\
\hline 1.0274 & 1.0749 & {$[62]$} & {$[63]$} & 4.99 \\
0.62151 & 0.6523 & 1.0809 & 1.0837 & 8.080 \\
0.43832 & 0.4356 & 0.6519 & 0.6536 & 10.77 \\
0.26122 & 0.2645 & 0.4350 & 0.4331 & 14.65 \\
0.17075 & 0.1751 & 0.1748 & 0.2609 & 18.51 \\
0.11963 & 0.1239 & 0.1237 & 0.1714 & 22.35 \\
0.08856 & 0.0917 & 0.0914 & 0.1191 & 26.22 \\
0.06918 & 0.0707 & 0.0705 & 0.0889 & 30.04 \\
0.05568 & 0.0561 & 0.0560 & 0.0554 & 33.85 \\
0.04488 & 0.0453 & 0.0451 & 0.0449 & 37.81 \\
\hline
\end{tabular}


Table 2. Comparative evaluation of the mechanical stability of graphene sheet compressed biaxially compared to the FSDT-DQM [62] and MD [63]. $\mu=1.81 \mathrm{~nm}^{2}, k_{s}=5 / 6, v=0.16, E=1 T P a$.

\begin{tabular}{cccc}
\hline \multicolumn{4}{c}{ Stability load $(n N / n m)$} \\
\hline OVFSDT & $\begin{array}{c}\text { FSDT-DQM } \\
{[62]}\end{array}$ & MD results [63] & $L_{x} / L_{y}$ \\
\hline 0.52449 & 0.5115 & 0.5101 & 0.5 \\
0.56223 & 0.5715 & 0.5693 & 0.75 \\
0.64225 & 0.6622 & 0.6595 & 1.25 \\
0.75576 & 0.7773 & 0.7741 & 1.5 \\
1.0134 & 1.0222 & 1.0183 & 1.75 \\
1.1703 & 1.1349 & 1.1297 & 2 \\
\hline
\end{tabular}

Table 3. Mechanical properties of graphene sheets.

$$
\begin{array}{cc}
\text { Isotropic } & E=1 \mathrm{TPa}, v=0.3 \\
\text { graphene sheet } & h=0.34 \mathrm{~nm}, \mathrm{Lx}=\mathrm{Ly}=10.2 \mathrm{~nm}, \\
{[8,56]} & \rho=2250 \mathrm{~kg} / \mathrm{m}^{3}, \alpha=1.1 \mathrm{e}-6 \mathrm{~K}
\end{array}
$$

To consider several cases of nonlocality against the transverse harmonic load, Figs. 2 and 3 are displayed. First of all, the higher-order nonlocal parameter has been investigated with the help of different dynamic loading conditions. As it is observed, the dynamic deflections decreased for increasing higher-order nonlocalities. Fig. 3 shows the conditions of lowerorder NSGT by changing the lower-order nonlocal parameter. It is worth noticing that an increased value for the higher-order nonlocal parameter has an increased impact on the dynamic deflection, than the lower-order nonlocal parameter (please, compare the plots of Figs. 2 and 3)

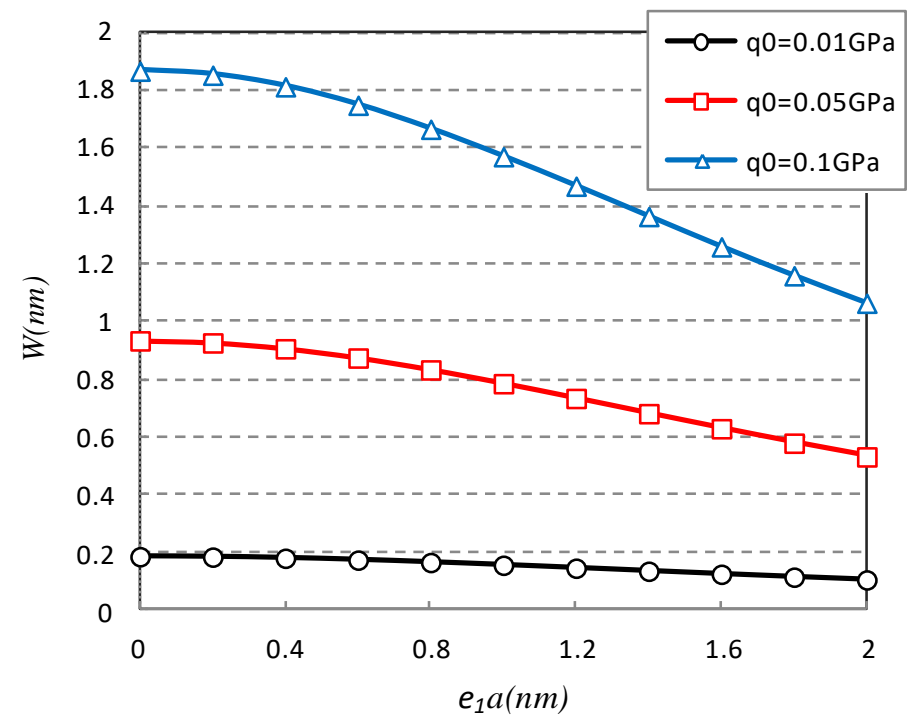

Fig.2. Transverse dynamic load vs. the higher-order nonlocal parameter. $e_{0} a=0.2 \mathrm{~nm}, l=0.5 \mathrm{~h}, m=n=1$, $\Delta r=0.1, \Delta T=200 K, x_{0}=y_{0}=0.5 L_{x}, c_{1}=c_{2}=L_{x}$. 


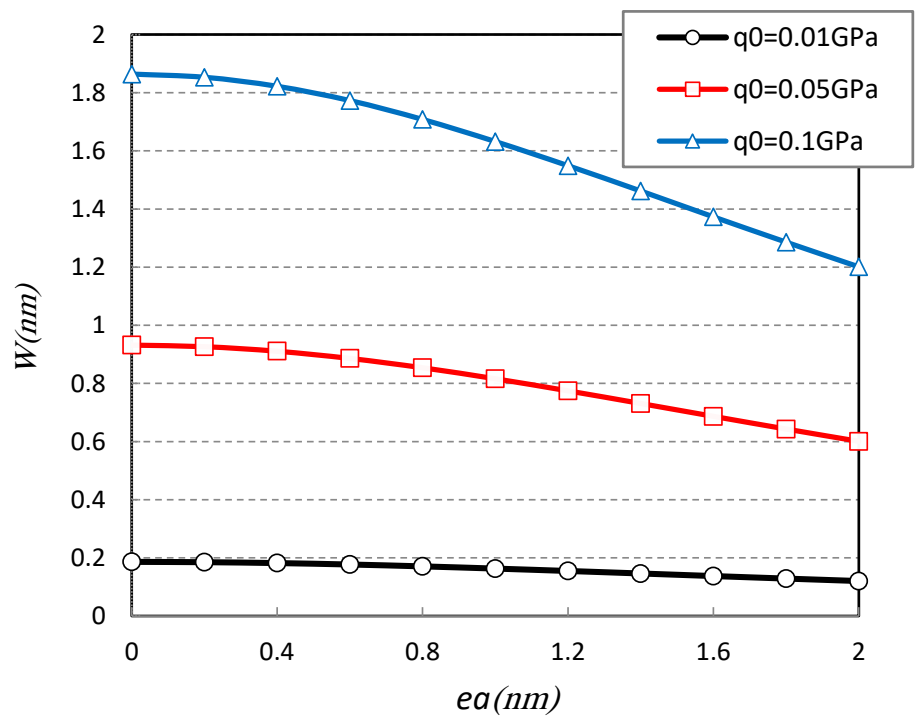

Fig.3. Transverse dynamic load vs. the nonlocal parameter. $e_{0} a=e_{1} a=e a, l=0.5 h, \Delta r=0.1, m=n=1$,

$$
\Delta T=200 K, x_{0}=y_{0}=0.5 L_{x}, c_{1}=c_{2}=L_{x} .
$$

Fig. 4 considers a higher-order NSGT for the graphene sheet under different excitation frequencies, and varying input geometrical parameters. As can be observed by these figures, the resonance situation occurs when $\Delta r=1$. Moreover, it is worth observing that after reaching the resonance condition, the dynamic deflections become smaller than those obtained before this resonance region. Naturally, if a large value of $\Delta r$ is assumed, the deflections cannot be significant, namely the system cannot have a vibrational response. It is interesting to note that a reduced distribution region for the transverse harmonic load, reduces the deformability of the structure. Furthermore, by comparing Fig. 4 and 5, we can observe that a farther distance of the centroid in the loaded region, yields meaningless deflections in the structure.

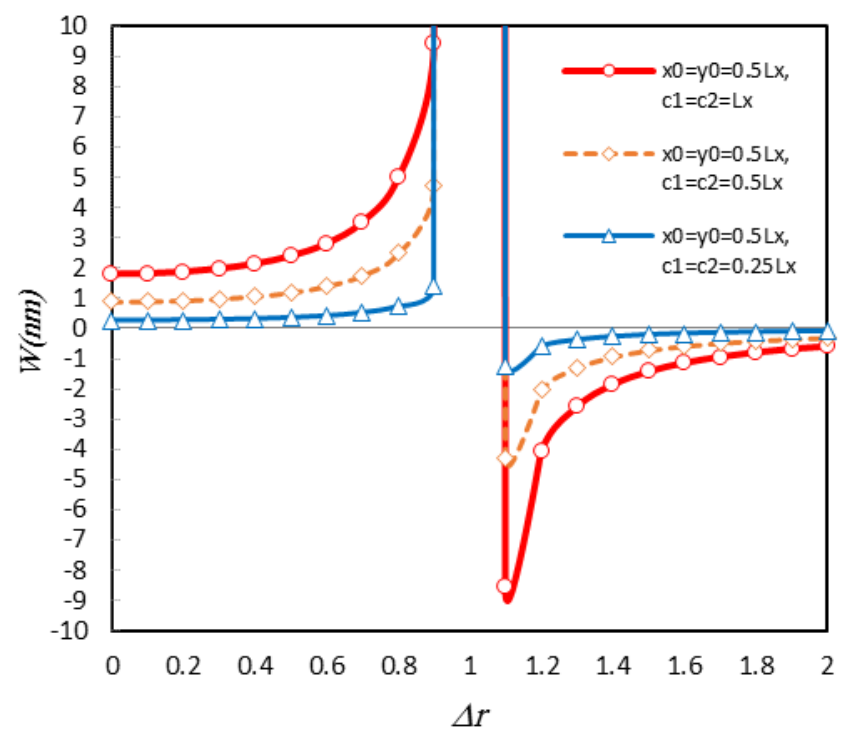

Fig.4. Distributed loads vs. the frequency ratio. $e_{0} a=0.2 \mathrm{~nm}, e_{1} a=0.4 \mathrm{~nm}, l=0.5 \mathrm{~h}, q_{0}=0.1 \mathrm{GPa}$, $\Delta T=200 K$. 


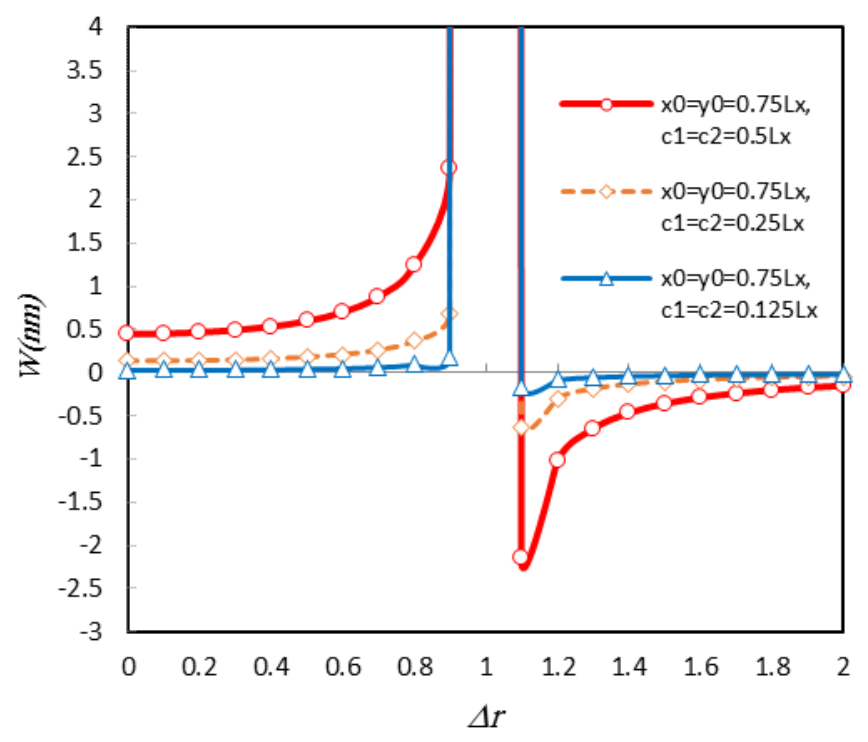

Fig.5. Distributed loads vs. the frequency ratio. $e_{0} a=0.2 \mathrm{~nm}, e_{1} a=0.4 \mathrm{~nm}, l=0.5 \mathrm{~h}, q_{0}=0.1 \mathrm{GPa}$,

$$
\Delta T=200 K \text {. }
$$

Fig. 6 plots the displacement response of the nanoplate vs. the temperature, while keeping constant the following input parameters: $e_{0} a=1 \mathrm{~nm}, e_{1} a=0.5 \mathrm{~nm}$, and $e_{0} a=0.5 \mathrm{~nm}, e_{1} a=1 \mathrm{~nm}$. Based on a comparative evaluation of the results, the nonlocal input parameters affect significantly the structural response, especially for a high temperature conditions. These results confirm the great impact of higher-order NSGT on the mechanical behavior of graphene sheets.

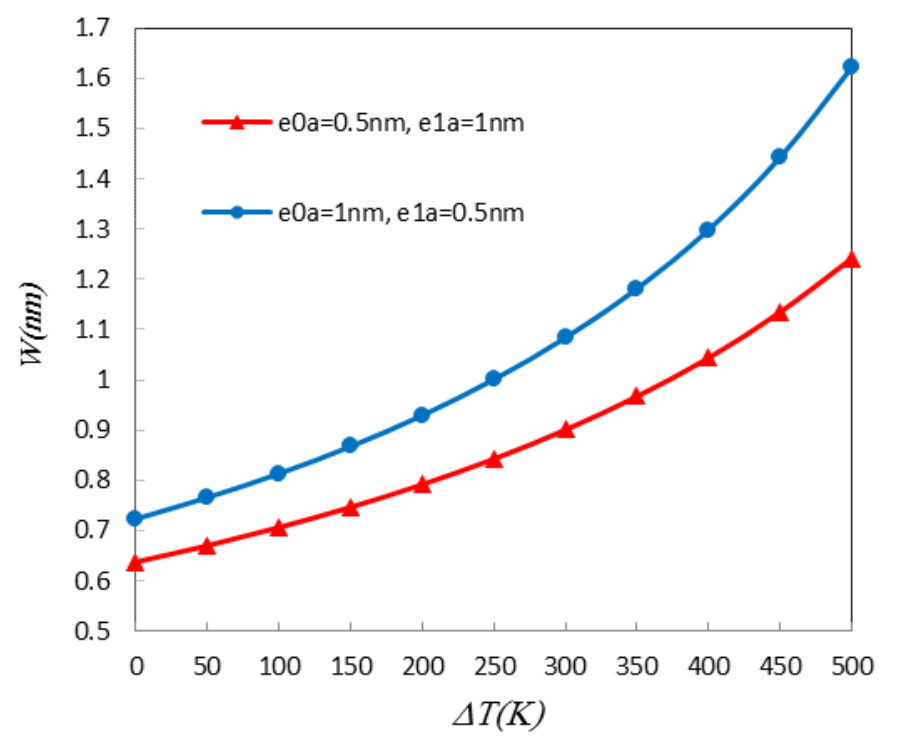

Fig.6. Higher and lower-order NSGT vs. the temperature variation. $l=0.5 h, q_{0}=0.05 G P a, \Delta r=0.1$, $m=n=1, x_{0}=y_{0}=0.5 L_{x}, c_{1}=c_{2}=L_{x}$.

\section{Conclusions}

This article has investigated the thermo-forced vibration of graphene nanoplates, subjected to a transverse dynamic loading applied both uniformly and harmonically. A new plate approach is here proposed to determine the equations of motion, whereas the higher-order NSGT is 
applied to evaluate the impacts at small scales. In addition, an analytic solution is here employed to check for the structural response. Based on a large parametric investigation, the following findings can be summarized as follows:

$\checkmark \quad$ The higher-order nonlocal parameter affects more significantly the structural response than other small scale parameters.

$\checkmark \quad$ A reduced sensitivity of the dynamic deflection when using higher-order nonlocality factors, within a higher-order NSGT context. This sensitivity of the response is less remarkable when a lower-order nonlocal parameter is applied.

$\checkmark \quad$ An increased value of the temperature emphasize the main differences in the structural results based on higher-order and lower-order nonlocal parameters. This means that, for high room temperatures, the higher-order nonlocal parameter play a key role.

\section{References}

[1] Taghioskoui, M., Trends in graphene research. Materials Today, 12, 34-37, 2009.

[2] Park, S., Ruoff, R. S., Chemical methods for the production of graphenes. Nature Nanotechnoly, 4, 217-224, 2009.

[3] An, X., Yu, J. C., Graphene-based photocatalytic composites. Royal Society of Chemistry Advances, 1, 1426-1434, 2011.

[4] Xiang, Q., Yu, J., Jaroniec, M., Graphene-based semiconductor photocatalysts. Chemical Society Reviews, 41, 782-796, 2012.

[5] Esmaeili, A., Entezari, M. H., Facile and fast synthesis of graphene oxide nanosheets via bath ultrasonic Irradiation. Journal of Colloid and Interface Science, 432, 19-25, 2014.

[6] Ebrahimi, F., Barati, M. R., Damping vibration analysis of graphene sheets on viscoelastic medium incorporating hygro-thermal effects employing nonlocal strain gradient theory. Composite Structures, 185, 241-253, 2018.

[7] Radic, N., Jeremić, D., Thermal buckling of double-layered graphene sheets embedded in an elastic medium with various boundary conditions using a nonlocal new first-order shear deformation theory. Composites Part B: Engineering, 97, 201-215, 2016.

[8] Malikan, M., Jabbarzadeh, M., Sh. Dastjerdi, Non-linear Static stability of bi-layer carbon nanosheets resting on an elastic matrix under various types of in-plane shearing loads in thermo-elasticity using nonlocal continuum. Microsystem Technologies, 23, 2973-2991, 2017.

[9] Malikan, M., Buckling analysis of a micro composite plate with nano coating based on the modified couple stress theory. Journal of Applied and Computational Mechanics, 4, 1$15,2018$.

[10] Malikan, M., Analytical predictions for the buckling of a nanoplate subjected to nonuniform compression based on the four-variable plate theory. Journal of Applied and Computational Mechanics, 3, 218-228, 2017. 
[11] Shahsavari, D., Karami, B., Mansouri, S., Shear buckling of single layer graphene sheets in hygrothermal environment resting on elastic foundation based on different nonlocal strain gradient theories. European Journal of Mechanics / A Solids, 67, 200-214, 2018.

[12] Farajpour, A., Haeri Yazdi, M. R., Rastgoo, A., Mohammadi, M., A higher-order nonlocal strain gradient plate model for buckling of orthotropic nanoplates in thermal environment. Acta Mechanica, 227, 1849-1867, 2016.

[13] Zhang, L. W., Zhang, Y., Liew, K. M., Vibration analysis of quadrilateral graphene sheets subjected to an inplane magnetic field based on nonlocal elasticity theory. Composites Part B: Engineering, 118, 96-103, 2017.

[14] Feng, C., Kitipornchai, S., Yang, J., Nonlinear free vibration of functionally graded polymer composite beams reinforced with graphene nanoplatelets (GPLs). Engineering Structures, 140, 110-119, 2017.

[15] Gholami, R., Ansari, R., Nonlinear harmonically excited vibration of third-order shear deformable functionally graded graphene platelet-reinforced composite rectangular plates. Engineering Structures, 156, 197-209, 2018.

[16] Civalek, O., The determination of frequencies of laminated conical shells via the discrete singular convolution method. Journal of Mechanics of Materials and Structures, 1, 163$182,2006$.

[17] Shen, J., Wang, H., Zheng, Sh., Size-dependent pull-in analysis of a composite laminated micro-beam actuated by electrostatic and piezoelectric forces: Generalized differential quadrature method. International Journal of Mechanical Sciences, 135, 353-361, 2018.

[18] Gürses, M., Civalek, O., Korkmaz, A., Ersoy H. Free vibration analysis of symmetric laminated skew plates by discrete singular convolution technique based on first-order shear deformation theory. International journal for numerical methods in engineering, 79, 290-313, 2009.

[19] Civalek, O., Nonlinear dynamic response of laminated plates resting on nonlinear elastic foundations by the discrete singular convolution-differential quadrature coupled approaches. Composites Part B: Engineering, 50, 171-179, 2013.

[20] Baltacioglu, A. K., Akgoz, B., Civalek, O., Nonlinear static response of laminated composite plates by discrete singular convolution method. Composite Structures, 93, 153-161, 2010.

[21] Civalek, O., Analysis of thick rectangular plates with symmetric cross-ply laminates based on first-order shear deformation theory. Journal of Composite Materials, 42, 2853-2867, 2008.

[22] Baltacioglu, A. K., Civalek, O., Akgoz, B., Demir, F., Large deflection analysis of laminated composite plates resting on nonlinear elastic foundations by the method of discrete singular convolution. International Journal of Pressure Vessels and Piping, 88, 290-300, 2011. 
[23] Shahba, A., Attarnejada, R., Hajilar, S. Free vibration and stability of axially functionally graded tapered Euler-Bernoulli beams. Shock and Vibration, 18, 683-696, 2011.

[24] Civalek, O., Demir, C., Buckling and bending analyses of cantilever carbon nanotubes using the Euler Bernoulli beam theory based on non-local continuum model. Asian Journal of Civil Engineering, 12, 651-661, 2011.

[25] Akgöz, B., Civalek, O., Buckling analysis of cantilever carbon nanotubes using the strain gradient elasticity and modified couple stress theories. Journal of Computational and Theoretical Nanoscience, 8, 1821-1827, 2011.

[26] Civalek, O., Free vibration of carbon nanotubes reinforced (CNTR) and functionally graded shells and plates based on FSDT via discrete singular convolution method. Composites Part B: Engineering, 111, 45-59, 2017.

[27] Malikan, M., Electroelastic biaxial compression of nanoplates considering piezoelectric effects, Journal of Polymer Science and Engineering, (2018),

http://dx.doi.org/10.24294/jpse.v0i0.558

[28] Malikan, M., Electro-mechanical shear buckling of piezoelectric nanoplate using modified couple stress theory based on simplified first order shear deformation theory. Applied Mathematical Modelling, 48, 196-207, 2017.

[29] Malikan, M., Tornabene, F., Dimitri, R., Nonlocal three-dimensional theory of elasticity for buckling behavior of functionally graded porous nanoplates using volume integrals. Materials Research Express, 5, 095006, 2018.

[30] Malikan, M., Electro-thermal buckling of elastically supported double-layered piezoelectric nanoplates affected by an external electric voltage. Multidiscipline Modeling in Materials and Structures, (2018), https://doi.org/10.1108/MMMS-012018-0010.

[31] Golmakani, M. E., Malikan, M., Sadraee Far, M. N., Majidi, H. R., Bending and buckling formulation of graphene sheets based on nonlocal simple first order shear deformation theory. Materials Research Express, 5, 065010, 2018.

[32] Malikan, M., Temperature influences on shear stability a nanosize plate with piezoelectricity effect. Multidiscipline modeling in materials and structures, 14, 125142, 2018.

[33] Wan, J., Chen, W. J., Li, X. P., Size-dependent free vibration analysis of composite laminated Timoshenko beam based on new modified couple stress theory. Archive of Applied Mechanics, 83, 431-444, 2013.

[34] Mercan, K., Civalek, O., DSC method for buckling analysis of boron nitride nanotube (BNNT) surrounded by an elastic matrix. Composite Structures, 143, 300-309, 2016.

[35] Demir, C., Mercan K, Civalek, O. Determination of critical buckling loads of isotropic, FGM and laminated truncated conical panel. Composites Part B: Engineering, 94, 1-10, 2016. 
[36] Mercan, K., Civalek, O., Buckling analysis of Silicon carbide nanotubes (SiCNTs) with surface effect and nonlocal elasticity using the method of HDQ. Composites Part B: Engineering, 114, 34-45, 2017.

[37] Jouneghani, F. Z., Dimitri, R., Bacciocchi, M., Tornabene, F., Free vibration analysis of functionally graded porous doubly-curved shells based on the first-order shear deformation theory. Applied Sciences, 7, 1-20, 2017.

[38] Nejati, M., Dimitri, R., Tornabene, F., Yas, M. H., Thermal buckling of nanocomposite stiffened cylindrical shells reinforced by Functionally Graded Wavy Carbon NanoTubes with temperature-dependent properties. Applied Sciences, 7, 1-24, 2017.

[39] Kiani, Y., Dimitri, R., Tornabene, F., Free vibration study of composite conical panels reinforced with FG-CNTs. Engineering Structures, 172, 472-482, 2018.

[40] Kiani, Y., Dimitri, R., Tornabene, F., Free Vibration of FG-CNT Reinforced Composite Skew Cylindrical Shells using Chebyshev-Ritz Formulation. Composites Part B: Engineering, 147, 169-177, 2018.

[41] Jouneghani, F. Z., Dimitri, R., Tornabene, F., Structural response of porous FG nanobeams under hygro-thermo-mechanical loadings. Composites Part B: Engineering, 152, 71-78, 2018.

[42] Fantuzzi, N., Tornabene, F., Bacciocchi, M., Dimitri, R., Free vibration analysis of arbitrarily shaped Functionally Graded Carbon Nanotube-reinforced plates. Composites Part B: Engineering, 115, 384-408, 2017.

[43] Nejati, M., Asanjarani, A., Dimitri, R., Tornabene, F., Static and free vibration analysis of functionally graded conical shells reinforced by carbon nanotubes. International Journal of Mechanical Sciences, 130, 383-398, 2017.

[44] Tornabene, F., Fantuzzi, N., Bacciocchi, M., Linear static response of nanocomposite plates and shells reinforced by agglomerated carbon nanotubes. Composites Part B: Engineering, 115, 449-476, 2017.

[45] Tornabene, F., Bacciocchi, M., Fantuzzi, N., Reddy, J. N., Multiscale approach for threePhase CNT/Polymer/Fiber laminated nanocomposite structures. Polymer Composite, In Press, DOI: $10.1002 /$ pc.24520.

[46] She, G. L., Yuan, F. G., Ren, Y. R., Thermal buckling and post-buckling analysis of functionally graded beams based on a general higher-order shear deformation theory. Applied Mathematical Modelling, 47, 340-357, 2017.

[47] She, G. L., Yuan, F. G., Ren, Y. R., Liu, H. B., Xiao, W. Sh., Nonlinear bending and vibration analysis of functionally graded porous tubes via a nonlocal strain gradient theory. Composite Structures, 203, 614-623, 2018.

[48] She, G. L., Yuan, F. G., Ren, Y. R., On wave propagation of porous nanotubes. International Journal of Engineering Science, 130, 62-74, 2018. 
[49] She, G. L., Ren, Y. R., Yuan, F. G., Xiao, W. S., On vibrations of porous nanotubes. International Journal of Engineering Science, 125, 23-35, 2018.

[50] She, G. L., Ren, Y. R., Xiao, W. S., Liu, H. B., Study on thermal buckling and postbuckling behaviors of fgm tubes resting on elastic foundations. Structural Engineering \& Mechanics, 66, 729-736, 2018.

[51] She, G. L., Yuan, F. G., Ren, Y. R., Xiao, W. S., On buckling and post-buckling behavior of nanotubes. International Journal of Engineering Science, 121, 130-142, 2017.

[52] Malikan, M., Nguyen, V. B., Tornabene, F., Electromagnetic forced vibrations of composite nanoplates using nonlocal strain gradient theory. Materials Research Express, 5, 075031, 2018.

[53] Malikan, M., Dimitri, R., Tornabene, F., Transient response of oscillated carbon nanotubes with an internal and external damping. Composites Part B: Engineering, 158, 198-205, 2019.

[54] Malikan, M., Nguyen, V. B., A novel one variable first-order shear deformation theory for biaxial buckling of a size-dependent plate based on the Eringen's nonlocal differential law. World Journal of Engineering, 2018. https://doi.org/10.1108/WJE-112017-0357

[55] Malikan, M., Nguyen, V. B., Tornabene, F., Damped forced vibration analysis of singlewalled carbon nanotubes resting on viscoelastic foundation in thermal environment using nonlocal strain gradient theory. Engineering Science and Technology, an International Journal, 21, 778-786, 2018.

[56] Malikan, M., Dimitri, R., Tornabene, F., Effect of sinusoidal corrugated geometries on the vibrational response of viscoelastic nanoplates. Applied Sciences, 8, 1432, 2018.

[57] Malikan, M., On the buckling response of axially pressurized nanotubes based on a novel nonlocal beam theory. Journal of Applied and Computational Mechanics, (2018), DOI: 10.22055/JACM.2018.25507.1274

[58] Malikan, M., Nguyen, V. B., Buckling analysis of piezo-magnetoelectric nanoplates in hygrothermal environment based on a novel one variable plate theory combining with higher-order nonlocal strain gradient theory. Physica E: Low-dimensional Systems and Nanostructures, 102, 8-28, 2018.

[59] Malikan, M., Dastjerdi, Sh., Analytical buckling of FG nanobeams on the basis of a new one variable first-order shear deformation beam theory. International Journal of Engineering \& Applied Sciences, 10, 21-34, 2018.

[60] Malikan, M., Sadraee Far, M. N., Differential quadrature method for dynamic buckling of graphene sheet coupled by a viscoelastic medium using neperian frequency based on nonlocal elasticity theory. Journal of Applied and Computational Mechanics. 4, 147$160,2018$. 
[61] Golmakani, M. E., Rezatalab, J., Nonuniform biaxial buckling of orthotropic Nano plates embedded in an elastic medium based on nonlocal Mindlin plate theory. Composite Structures, 119, 238-250, 2015.

[62] Golmakani, M. E., Sadraee Far, M. N., Buckling analysis of biaxially compressed double-layered graphene sheets with various boundary conditions based on nonlocal elasticity theory. Microsystem Technologies, 23, 2145-2161, 2017.

[63] Ansari, R., Sahmani, S., Prediction of biaxial buckling behavior of single-layered graphene sheets based on nonlocal plate models and molecular dynamics simulations. Applied Mathematical Modelling, 37, 7338-51, 2013. 